\title{
Effects of Secondhand Smoke Exposure and Noise Exposure on Tinnitus Occurrence in College Students and Adolescents
}

\author{
Laurel Elise Money ${ }^{1}$ Ishara Ramkissoon ${ }^{1,2}$ \\ ${ }^{1}$ University of South Alabama, Mobile, Alabama \\ 2 University of Kwa-Zulu Natal, Durban, South Africa \\ J Am Acad Audiol 2020;31:286-291.
}

Address for correspondence Ishara Ramkissoon, University of South Alabama, Mobile, AL 36688 (e-mail: ramkissoon@southalabama.edu).

\begin{abstract}
Keywords

- adolescents

- college students

- noise

- secondhand smoke

- tinnitus

Background Recent research indicated that young adults and adolescents reported tinnitus in higher numbers than previously. Thus, it is important to fully investigate risk factors for tinnitus in adolescents and young adults.

Purpose The current study examined the influence of two environmental risk factors, secondhand smoke (SHS) exposure and noise exposure on tinnitus occurrence as self-reported by U.S. adolescents and young adults.

Research Design A nonexperimental, cross-sectional design was used for this survey study.

Study Sample Of 265 surveys received, 43 were excluded due to ineligibility. The remaining 222 surveys constituted the study sample. Participant respondents included 80 high school students (ages 14-17) and 142 college students (ages 18-30). The sample was primarily female $(n=160)$.

Data Collection and Analysis Three (3) yes/no survey questions regarding SHS exposure, noise exposure, and tinnitus occurrence were analyzed. Statistical analyses included logistic regression, chi-square follow-up tests, and Pearson bivariate correlation analysis.

Results Results revealed that $40 \%$ of young adults and adolescents surveyed reported that they experienced tinnitus. Regression analysis revealed significant main effects for noise $(p=0.004)$, gender $(p=0.017)$, and the interaction of SHS and noise $(p=0.001)$. There was no main effect of SHS exposure nor age on tinnitus occurrence. Follow-up chisquare analysis conducted to probe the gender effect revealed that females $(45.1 \%)$ were more likely to experience tinnitus than males (27.7\%). Chi-square testing to examine the significant interaction effect revealed statistical significance $(p=0.001)$ for individuals with reported noise exposure but not for individuals without noise exposure. In the noiseexposed group, individuals who also reported SHS exposure had a lower occurrence of tinnitus (23.6\%). In contrast, the noise-exposed individuals without SHS exposure had a higher prevalence of tinnitus (57.9\%). An additional chi-square follow-up analysis to examine the main effect of noise revealed no significance $(p=0.199)$. However, there was a significant $(p<0.01)$ negative correlation $(r=-0.244)$ of noise exposure with age. Conclusion The current study results suggest there is a higher than expected report of tinnitus incidence in adolescents and young adults. The relationship between tinnitus occurrence and a combined exposure to noise and secondhand smoke revealed a unique effect in adolescents and young adults.
\end{abstract}

Copyright $\odot 2020$ by the American Academy of Audiology. All rights reserved. Thieme Medical Publishers, Inc., 333 Seventh Avenue, New York, NY 10001, USA. Tel: +1(212) 760-0888
DOI https://doi.org/ 10.3766/jaaa.19032. ISSN 1050-0545. 


\section{Introduction}

Tinnitus is most often described as a ringing or buzzing in one's ears without the presence of external auditory stimulation. It can create difficulties with concentration, inability to hear sounds of interest, and frustration for those experiencing it. It is typically associated with older adults, with approximately $13-33 \%$ of individuals $>60$ years reporting tinnitus (McCormack et al, $2016^{12}$ ). However, recent studies indicated young adults and teenagers may be experiencing tinnitus in higher numbers than previously believed. For example, Muhr and Rosenhall $(2010)^{14}$ reported that $23 \%$ of college-aged individuals have experienced tinnitus, and Bhatt $(2017)^{2}$ reported that $59.3 \%$ of college-aged individuals had experienced tinnitus at least once in their lifetime. These reports indicated prevalence discrepancies, and it may be unclear which age group typically experiences more tinnitus. Although tinnitus (a symptom) is more commonly reported in those with audiometric hearing loss (Davis and El Refaie, 20004), it does occur in individuals with normal hearing thresholds. For example, one study on participants aged 12-19 years ( $\mathrm{n}=4,396$ ) found that $23.6 \%$ of those who reported tinnitus also had some hearing loss, but $76.4 \%$ of those who reported tinnitus had normal hearing thresholds (Mahboubi et al, $\left.2013^{10}\right)$. Thus, tinnitus in the presence of normal hearing is not unexpected among adolescents. In addition, the interaction of age with gender influences tinnitus prevalence; in children, females appear to have a higher likelihood of developing tinnitus (Lee and Kim, $2018^{9}$ ) but in adults, males have a higher prevalence of tinnitus (McCormack et al, 2016 ${ }^{12}$ ).

The etiology of tinnitus is often unknown (Stouffer and Tyler, $1990^{20}$ ). Tinnitus can occur when microscopic outer hair cells in the cochlea are damaged leading to abnormal movement with electrical impulses sent to the brain that are perceived as noise or tinnitus. Several other variables, including age, gender, socioeconomic status, occupation, smoking, and alcohol, are related to the prevalence of tinnitus (Davis and El Refaie, 2000 ${ }^{4}$ ). In addition, other factors, including excessive noise, have been studied thoroughly and are well known to be associated with tinnitus (Moore et al, 2016; ${ }^{13}$ Bhatt, 2017²).

\section{Exposure to Noise}

Permanent damage due to excessive noise exposure can be hazardous for college students who are often exposed to recreational noise, more often than older generations. Students can be exposed to excessive noise from workplace settings, music at concerts, in bars, or from personal music players. Mahboubi et al $(2013)^{10}$ studied the association between tinnitus and noise (music) exposure in normal hearing adolescents aged $12-19$ years by examining the odds ratio (OR). Their results indicated that adolescents who were exposed to recreational noise had a significantly higher odds ofreporting chronic tinnitus (OR: 2.08; 95\% confidence interval [CI]: 1.353 .18 ) or overall tinnitus (OR: 2.17 ; 95\% CI: $1.30-3.62$ ). In a Swedish study of healthy young men $(\mathrm{n}=839)$ aged $19-22$ years, $23 \%$ of participants reported experiencing tinnitus (Muhr and Rosenhall, 2010 ${ }^{14}$ ). In addition, participants who reported playing loud music had a significantly higher preva- lence of tinnitus compared with the control group $(p \leq 0.001)$, and one in ten participants reported experiencing tinnitus "often" after exposure to noise. In a study of college students $(\mathrm{n}=238)$, Rawool and Colligon-Wayne $(2008)^{19}$ reported that young adults' attitudes toward noise could have negative impacts on their hearing because $75 \%$ of students surveyed knew that noise could cause hearing loss, but $50 \%$ still knowingly exposed themselves to dangerous noise levels. This perceived invulnerability was evidenced by the fact that $76 \%$ of college students surveyed believed they would not lose their hearing until they were older, and $58 \%$ believed that they did not need to worry about the ringing in their ears as long as it was temporary.

\section{Exposure to Secondhand Smoke (SHS)}

The effect of cigarette smoking on the auditory system has been recently documented (c.f., Ramkissoon and Chambers, $2008 ;^{18}$ Harkrider et al, 2001; ${ }^{6}$ Bhatt, 2017²). Effects of SHS exposure, however, is less studied. SHS exposure or passive smoking occurs when a person involuntarily inhales smoke from cigarettes typically being smoked by another person. Cigarette smoke has $>4,000$ chemicals and 50 carcinogens, and exposure to SHS is associated with a plethora of health problems, including heart disease, lung cancer, and respiratory infections (National Cancer Institute, $1997^{15}$ ). A study using data from the National Health and Nutrition Examination Survey (2013-2014 cycle) found that 58 million (25\%) US nonsmokers were exposed to SHS (Tsai et al, 2018 ${ }^{21}$ ). In addition, SHS was recently associated with hearing loss (Fabry et al, 2011; ${ }^{5}$ Lalwani et al, $2011^{8}$ ). Given that 29 million US adults experience hearing loss, SHS could well be a primary or contributory cause of hearing loss nationally.

College students are particularly vulnerable to SHS exposure because of their socialization patterns on campus, at bars/clubs, or in homes and cars with friends. In fact, as many as $83 \%$ of students reported at least one exposure to SHS in the last seven days (Wolfson et al, 200922). Specifically, 65\% of SHS-exposed students reported exposure at a restaurant/bar, $55 \%$ in the same room with a smoker, and $38 \%$ in a car with a smoker.

One study examined SHS in former smokers and never smokers aged 20-65 years and found that $46.6 \%$ of SHSexposed former smokers along with $26.6 \%$ of never smokers experienced high-frequency hearing loss (Fabry et al, 20115). They reported a significant positive association between SHS exposure and hearing loss at low/mid frequency levels for both former and never smoker groups. In former smokers, SHS exposure and high-frequency hearing loss were significantly and positively related (Fabry et $\mathrm{al}, 2011^{5}$ ). These results were some of the first to connect SHS exposure to hearing loss. More recently, Chang et al $(2015)^{3}$ examined smoking effects on hearing loss in Korean adults aged 19-80; one group was "passive smokers," defined as people who had never smoked but were exposed to SHS daily. These passive smokers had worsened pure-tone hearing thresholds than nonsmokers without SHS exposure.

Lalwani et al $(2011)^{8}$ examined hearing status in 1,533 adolescents aged $12-19$ years, with $49 \%$ of the sample having SHS exposure. They defined hearing loss as pure-tone 
thresholds $>15 \mathrm{~dB}$ and categorized hearing loss by laterality and frequency range involved. They found that prevalence of sensorineural hearing loss was higher in adolescents with SHS exposure compared with unexposed individuals, for all hearing loss categories. In particular, SHS-exposed adolescents had significantly elevated hearing thresholds for unilateral low frequency hearing loss $(p=0.03)$ and bilateral high-frequency hearing loss $(p=0.04)$. The relationship between SHS exposure and hearing thresholds at individual frequencies was also examined. Thresholds in SHS-exposed adolescents were significantly elevated at 2000,3000, and $4000 \mathrm{~Hz}$ compared with the nonexposed group. Notably, these statistically significant differences in hearing thresholds were on the order of 1-3 dB; this has minimal clinical significance.

The impact of SHS exposure on auditory function was further explored with pure-tone audiometry and evoked potential testing in college students aged 18-23 (Ramkissoon and Batavia, $2018^{17}$ ). Their results revealed that although all participants had normal hearing thresholds, central auditory system functioning was affected in students exposed to SHS. Specifically, young adults with SHS exposure had delayed wave $\mathrm{Pb}$ latency (auditory middle latency response) and decreased wave $\mathrm{V} / \mathrm{I}$ amplitude ratio (auditory brainstem response) compared with individuals who were unexposed to SHS. These findings suggested a possible toxicity effect of SHS exposure on the central auditory system.

Mahboubi et al $(2013)^{10}$ evaluated the occurrence of tinnitus in 4,396 adolescents (12-19 years); of whom, $19.4 \%$ were exposed to SHS. Results revealed that SHS-exposed adolescents were almost twice as likely ( $\mathrm{OR}=1.95,[\mathrm{Cl}: 1.23-3.11])$ to develop overall tinnitus and 1.76 (OR, CI: 1.00-3.10) times more likely to develop chronic tinnitus. Another study surveyed Serbian secondary school students aged $14-20$ years $(n=771)$ regarding tinnitus, SHS exposure, and noise exposure (Marmut et al, $2014^{11}$ ). In total, $12.8 \%$ of students reported tinnitus, with girls reporting it more frequently than boys. About $50 \%$ of students reported were being exposed to SHS. The Spearman's coefficient results revealed a positive significant correlation between tinnitus and smoking $(p=0.196)$, tinnitus and SHS exposure $(\rho=0.125)$, and daily duration of SHS exposure and tinnitus $(\rho=0.224)$ in boys. In girls, there was a significant positive relationship $(\rho=0.110)$ between tinnitus and daily time spent indoors being exposed to SHS. Multivariate logistic regression analysis revealed a significant independent effect of daily duration of SHS exposure to tinnitus $(p=0.009)$ in girls. This means that daily duration of SHS exposure predicted tinnitus occurrence in girls. This study strongly suggested that SHS exposure and tinnitus may be related.

\section{Purpose of the Present Investigation}

The goal of the current study was to examine how two environmental factors, SHS exposure and noise exposure, related to the occurrence of tinnitus in normal hearing US college students and adolescents. This study focused on a younger population to examine, in greater depth, the effects of SHS and noise on tinnitus occurrence because most previous research was conducted in adults, particularly older, hearing-impaired individuals. Although a few past studies have connected SHS exposure to hearing loss, there is limited information relating SHS to tinnitus. This is also the first known study to examine possible interactions between SHS exposure and noise exposure as it impacts tinnitus occurrence. The results may be useful for public health initiatives promoting hearing health, particularly in college students and adolescents.

\section{Research Questions}

- Is exposure to SHS related to tinnitus occurrence in college students and adolescents?

- Is exposure to noise related to tinnitus occurrence in college students and adolescents?

- Are there interactions between SHS exposure and noise exposure as they influence tinnitus occurrence in college students and adolescents?

\section{Methods}

\section{Study Design}

This study used a nonexperimental cross-sectional design. Data were gathered from an archived survey study approved by the Institutional Review Board protocol \#12-091. The original survey included questions about participants' biographical information, any hearing/ communication difficulties, exposure to SHS (e.g., number of smokers in the home), exposure to loud noises (e.g., type of noise environment), and tinnitus (e.g., which ear and how often). However, the current study approved under Institutional Review Board protocol \#17-200 analyzed a subset of the data. Three specific yes/no questions about the occurrence of noise exposure (Have you been exposed to loud noises on a regular basis?), SHS exposure (Do you live in a home where one or more people smoke tobacco products?), and tinnitus (Do you experience tinnitus [noise in your ear like ringing or buzzing]?) were analyzed.

\section{Participants}

A total of 265 surveys were available for this study (171 college students and 94 adolescents). Forty-three surveys were excluded from data analysis for reasons such as current smoking, age, medications linked to tinnitus, missing data, and hearing loss. Of the remaining 222 participants, 160 were female, eighty were high school students (aged 14-17 years), and 142 were college students (aged $18-30$ years). All participants were in overall good health with no reported hearing/communication difficulties. The mean age was 18 years overall (range: $14-30$ years, $S D=2.73$ ); 15 years for adolescents and 19 years for college students.

\section{Procedure}

Survey responses to the three selected questions for $n=222$ participants were entered into a spreadsheet.

Participants were assigned an ID number, and other information such as age, gender, smoking behavior, and reported communication difficulties were also entered into the spreadsheet. Original survey responses for SHS exposure at home was divided into two categories-"SHS in the home" and "SHS near the home." For analysis purposes, these two 
Table 1 Variables in the Regression Equation

\begin{tabular}{|l|l|l|l|}
\hline Factors & $\boldsymbol{\beta}$ & Standard Error & Significance \\
\hline Gender & 0.800 & 0.334 & $0.017^{*}$ \\
\hline Age & 0.005 & 0.054 & 0.929 \\
\hline SHS & 0.557 & 0.419 & 0.183 \\
\hline Noise & 1.020 & 0.358 & $0.004^{* *}$ \\
\hline $\begin{array}{l}\text { Interaction of } \\
\text { SHS and noise }\end{array}$ & -2.074 & 0.647 & $0.001^{* *}$ \\
\hline Constant & -1.369 & 1.076 & 0.254 \\
\hline
\end{tabular}

${ }^{*} p<0.05$.

${ }^{* *} p<0.01$.

responses were collapsed into one category titled "SHShome" and a "yes" response to either question was accepted. Data were transferred into SPSS version 22 (IBM Corp., Armonk, NY) for statistical analysis.

\section{Results}

The current investigation examined the relationship between reported SHS exposure, noise exposure, and tinnitus occurrence in college students and adolescents. Of 222 participants, $31 \%(\mathrm{n}=68)$ reported SHS exposure, $41 \%(\mathrm{n}=91)$ reported noise exposure, and $40 \%(\mathrm{n}=89)$ reported experiencing tinnitus.

The first statistical analysis conducted was a binary logistic regression to examine the relationship of SHS exposure, noise exposure, gender, and age on tinnitus occurrence. The results from the regression analysis are shown in - Table 1. There was a significant main effect on noise exposure $(p=0.004)$ and a significant interaction effect (SHS/Noise) on tinnitus $(p=0.001)$. Gender was also a significant factor in predicting tinnitus ( $p=0.017$ ). There was no main effect of SHS exposure nor age on tinnitus occurrence. The slope of -2.074 for the SHS/noise interaction variable had the highest predictive value in this analysis, that is, the SHS/noise interaction was the most likely independent variable to produce lower levels of tinnitus. The current standard error range of 0.05-0.6 indicated that there was less error in the measurement.

A chi-square analysis conducted to probe the gender effect revealed that females were more likely than males to experience tinnitus, with $45.1 \%$ of females reporting tinnitus and only $27.7 \%$ of males reporting tinnitus. Furthermore, chi-
Table 3 Correlations between Variables of Interest

\begin{tabular}{|l|l|l|l|l|}
\hline & $\begin{array}{l}\text { SHS } \\
\text { Exposure }\end{array}$ & $\begin{array}{l}\text { Noise } \\
\text { Exposure }\end{array}$ & Tinnitus & Age \\
\hline $\begin{array}{l}\text { SHS } \\
\text { exposure }\end{array}$ & 1 & 0.122 & -0.085 & 0.064 \\
\hline $\begin{array}{l}\text { Noise } \\
\text { exposure }\end{array}$ & 0.122 & 1 & 0.084 & $-0.244^{* *}$ \\
\hline Tinnitus & -0.085 & 0.084 & 1 & -0.028 \\
\hline Age & 0.064 & $-0.244^{* *}$ & -0.028 & 1 \\
\hline
\end{tabular}

${ }^{* *}$ Correlation is significant at the 0.01 level (two-tailed).

square analyses were conducted to examine the significant interaction effect (SHS by noise) using the "split file" feature in SPSS. The results are shown in $\mathbf{- T a b l e ~} \mathbf{2}$, which categorizes participants by noise exposure, that is, "no noise" and "noiseexposed." Within these two noise categories, participants were further divided by SHS exposure, and the resultant number of participants per category is shown with the percent of the total sample in parenthesis. The results indicated statistical significance $(p=0.001)$ for the group of individuals with noise exposure but not for individuals without noise exposure. In the noise-exposed group, individuals who also reported SHS exposure had a lower occurrence of tinnitus (23.6\%). By contrast, the noise-exposed individuals without SHS exposure had a higher prevalence of tinnitus (57.9\%). An additional chi-square follow-up analysis was conducted to examine the main effect of noise; this analysis did not reveal significance $(p=0.199)$. Chi-square analysis for the SHS exposure variable was also not significant.

Correlation analysis was conducted in SPSS to examine the relationship between tinnitus and SHS exposure, noise exposure, and age. The results in -Table 3 indicate that noise exposure was significantly correlated with age; this was a low negative correlation. No other bivariate correlations were significant.

\section{Discussion}

The current study hypothesized that noise exposure and SHS exposure would each predict reported tinnitus in college students and adolescents. In addition, it was hypothesized that individuals who experienced a combination of SHS exposure and noise exposure would be more likely to report an even higher occurrence of tinnitus. The current results did

Table 2 Tinnitus in Individuals Based on Noise Exposure and SHS Exposure

\begin{tabular}{|l|l|l|l|l|l|}
\hline Noise Group & & $\mathbf{n}(\%)$ & Tinnitus: $\mathbf{n}(\%)$ & \multicolumn{2}{l|}{ Significance } \\
\cline { 4 - 6 } & & & Absent & Present \\
\hline \multirow{2}{*}{ No noise } & No SHS & $97(44 \%)$ & $64(66.0 \%)$ & $33(34.0 \%)$ & 0.199 \\
\cline { 2 - 6 } & SHS-exposed & $34(14 \%)$ & $19(55.9 \%)$ & $15(44.1 \%)$ & $0.001^{*}$ \\
\hline Noise-exposed & No SHS & $57(26 \%)$ & $24(42.1 \%)$ & $33(57.9 \%)$ & $8(23.6 \%)$ \\
\cline { 2 - 5 } & SHS-exposed & $34(15 \%)$ & $26(76.4 \%)$ & & \\
\hline
\end{tabular}

*Fisher's exact test used for significance; $\mathrm{n}=222$. 
not support all hypotheses and revealed some unexpected findings. The discrepancies found in the current results compared with previous studies indicate that further research on current study variables as related to tinnitus should be conducted.

The hypothesis related to noise exposure was not supported. The initial regression analysis showed that noise exposure was a significant factor in predicting tinnitus. However, a follow-up chi-square test for significance indicated the relationship between tinnitus and noise exposure was not significant. This result differed from past studies. For example, Mahboubi et al $(2013)^{10}$ reported that tinnitus was exacerbated by exposure to excessive noise in adolescents, $12-19$ years, and Hinalaf et al $(2017)^{7}$ indicated that adolescents with higher levels of general music exposure had significantly higher reported tinnitus (72.41\%). The current study results might have differed from these past studies because of a smaller convenience sample and different test procedures used.

The current results indicated that SHS exposure was not significant in predicting tinnitus, a finding which differed from previous studies. For example, Marmut et al (2014) ${ }^{11}$ found that tinnitus occurrence was correlated with daily duration of SHS exposure in boys and girls aged 15-19 years and also with SHS exposure overall, in boys only. Similarly, Mahboubi et al $(2013)^{10}$ found that adolescents exposed to SHS were almost twice as likely to develop tinnitus and 1.76 times more likely to develop chronic tinnitus. However, the current study included design elements that might explain our findings being different. Specifically, there was a smaller proportion of males (23\%) in the current than in the past studies (36-43\%), and the current study included both adolescent and young adult participants compared with Marmut and Mahboubi who examined the link between SHS exposure and tinnitus occurrence in adolescents only. In addition, other studies reported a link between SHS exposure and sensorineural hearing loss in adolescents (Lalwani et al, $2011^{8}$ ) as well as the SHS exposure and central auditory function (auditory brainstem response, auditory middle latency response) in young adults (Ramkissoon and Batavia, $2018^{17}$ ). The lack of relationship between SHS exposure and tinnitus found in the current results compared with these previous studies indicates the need for further research to better understand this variable.

Perhaps, the most interesting finding in the current study was related to the interaction of SHS exposure and noise exposure on tinnitus occurrence. The results showed that individuals who reported exposure to both SHS and noise had the lowest tinnitus prevalence of $23 \%$ (refer to - Table 2 ). This was an unexpected result. The other known studies that evaluated both SHS and noise exposure as related to tinnitus were reported by Marmut et al $(2014)^{11}$ and Mahboubi et al $(2013)^{10}$. However, in both of those studies, each variable was analyzed separately, and the authors did not examine any interaction effect of SHS and noise in the same participant. Thus, there are no known past results to compare the current interaction effect with. We had anticipated a cumulative effect of SHS and noise exposure on tinnitus occurrence, but instead the lower occurrence of tinnitus in these individuals warrants further investigation.
A few other current results are interesting. First, the current prevalence of tinnitus in college students and adolescents was 40\%, much higher than past reports (Davis and El Refaie, 2000; Muhr and Rosenhall, 2010; ${ }^{14}$ Moore et al, $2016^{13}$ ) but similar to recent reports (Bhatt, 2017; ${ }^{2}$ Hinalaf et al, 2017 ${ }^{7}$ ) where $64 \%$ and $59.3 \%$ of college students reported occurrence of tinnitus, respectively. Overall, although the current results and these past studies did not all have the same design, the reported tinnitus prevalence of 40-64\% indicates that many younger adults and adolescents appear to be susceptible to tinnitus than previously believed. Another interesting finding in this study was the gender effect, with female gender being a significant factor in predicting tinnitus. A higher percentage of females reported tinnitus than males, which is similar to other studies with adolescents where female gender was a risk factor for tinnitus (Mahboubi et al, 2013; ${ }^{10}$ Marmut et al, 2014; ${ }^{11}$ Park et al, $2014^{16}$ ). Third, the current result (see - Table 2 ) indicated that among noise-exposed individuals $(\mathrm{n}=91)$, the highest occurrence of tinnitus ( $\mathrm{n}=33 ; 57.9 \%)$ occurred in individuals with no reported SHS exposure. This implied that having noise exposure without SHS exposure increases the chance of tinnitus. Perhaps, noise exposure is the most important predictor of tinnitus occurrence as shown previously (Muhr and Rosenhall, 2010; ${ }^{14}$ Mahboubi et al, 2013; ${ }^{10}$ Marmut et al, 2014; ${ }^{11}$ Lee and Kim, $2018^{9}$ ). Overall, however, the specific implications of this result are unclear and need further exploration. Finally, the significant negative correlation $(r=-0.244)$ between noise and age indicated that as age increased, the amount of noise exposure decreased. For the current sample, it may be assumed based on the mean age of 18.2 years $(S D=2.7)$ that individuals aged 21-30 years had less noise exposure, possibly because of changes in recreational and occupational environments, than those aged 14-20 years. This has potentially important clinical and counseling implications. An additional important application of the current results is related to educating adolescents and young adults about how noise is significantly linked to tinnitus.

A limitation of this study is that tinnitus occurrence was evaluated based on self-reported data without corroboration through clinical assessment. For the noise factor, our survey question recruited individuals with regular, consistent exposure to loud noise but did not specify the type, source, or specific duration of the noise exposure. Also, participants who reported any exposure to SHS, irrespective of SHS exposure duration, were included which may have influenced the results. Furthermore, SHS exposure was not classified by cotinine level, a more objective measure. However, Avila-Tang et al (2013) ${ }^{1}$ indicated that often even known SHS-exposed individuals tested negative for SHS exposure on objective measures. Follow-up research currently ongoing in our laboratory is examining details about noise and SHS exposure as it influences tinnitus occurrence and reported hearing difficulties.

\section{Conclusion}

The current study suggests there is a higher-than-expected report of tinnitus incidence in adolescents and young adults. This result supports other recent studies showing similar 
high tinnitus incidence in college students. The relationship between tinnitus occurrence and a combined exposure to noise and SHS revealed a unique effect. Specifically, noiseexposed individuals without SHS exposure had the highest tinnitus prevalence. This relationship needs further investigation.

\section{Abbreviations}

CI confidence interval

OR odds ratio

SHS secondhand smoke

\section{References}

1 Avila-Tang E, Elf JL, Cummings KM, Fong GT, Hovell MF, Klein JD, Mcmillen R, Winickoff JP, Samet JM. Assessing secondhand smoke exposure with reported measures. Tob Control 2013 22(03): 156-163

2 Bhatt I. Prevalence of and risk factors for tinnitus and tinnitusrelated handicap in a college-aged population. Ear Hear 2017;39 (03):517-526

3 Chang J, Ryou N, Jun J, Hwang S, Song J, Chae S. Effect of cigarette smoking and passive smoking on hearing impairment: data from a population-based study. PLoS One 2015;11(01):e0146608

4 Davis A, El Refaie A. Epidemiology of tinnitus. In: Tyler R, ed. Tinnitus Handbook. San Diego, CA: Singular Thomson Learning; $20001-21$

5 Fabry DA, Davila EP, Arheart KL, Serdar B, Dietz NA, Bandiera FC, Lee DJ. Secondhand smoke exposure and the risk of hearing loss. Tob Control 2011 20(01):82-85

6 Harkrider AW, Champlin CA, McFadden D. Acute effect of nicotine on non-smokers: I. OAEs and ABRs. Hear Res 2001;(160(12):73-88

7 Hinalaf M, Maggi AL, Hug MX, Kogan P, Villalobo JP, Biassoni EC. Tinnitus, medial olivocochlear system, and music exposure in adolescents. Noise Health 2017 19(87):95-102

8 Lalwani AK, Liu Y, Weitzman M. Secondhand smoke and sensorineural hearing loss in adolescents. Arch Otolaryngol Head Neck Surg 2011 137(07):655-662
9 Lee D, Kim Y. Risk factors of pediatric tinnitus: systematic review and meta-analysis. Laryngoscope 2018 128:1462-1468

10 Mahboubi H, Oliaei S, Kiumehr S, Dwabe S, Djalilian HR. The prevalence and characteristics of tinnitus in the youth population of the United States. Laryngoscope 2013 123(08):2001-2008

11 Marmut Z, Belojevic G, Backovic D, Zivojinovic J, Tomanic M, Hadzic E. Tinnitus among Serbian secondary school students in relation to their behavior and habits. Noise Health 2014;16(69): 73-78

12 McCormack A, Edmondson-Jones M, Somerset S, Hall D. A systematic review of the reporting of tinnitus prevalence and severity. Hear Res 2016 337:70-79

13 Moore D, Zobay O, Mackinnon R, Whitmer W, Akeroyd M. Lifetime leisure music exposure associated with increased frequency of tinnitus. Hear Res 2016 347:18-27

14 Muhr P, Rosenhall U. Self-assessed auditory symptoms, noise exposure, and measured auditory function among healthy young Swedish men. Int J Audiol 2010 49(04):317-325

15 National Cancer Institute. Health effects of exposure to environmental tobacco smoke: the report of the California Environmental Protection Agency. Smoking and Tobacco Control Monograph No. 10. Bethesda, MD: U.S. Department of Health and Human Services, National Institutes of Health; 1997

16 Park B, Choi H, Lee H, An S, Kim S, Lee J, Hong S, Kim H. Analysis of the prevalence of and risk factors for tinnitus in a young population. Otol Neurotol 2014 35:1218-1222

17 Ramkissoon I, Batavia M. Effect of secondhand smoke exposure on hearing and auditory evoked potentials in young adults. J Am Acad Audiol 2018 29(08):685-695

18 Ramkissoon I, Chambers RD. Effects of chronic and acute smoking on AMLRs in older and younger listeners. Int J Audiol 2008;47(12): 715-723

19 Rawool V, Colligon-Wayne L.. Auditory lifestyles and beliefs related to hearing loss among college students in the USA. Noise Health 2008 10(38):1-10

20 Stouffer JL, Tyler RS. Characterization of tinnitus by tinnitus patients. J Speech Hear Disord 1990 55(03):439-453

21 Tsai J, Homa D, Gentzke A, Mahoney M, Sharapova S, Sosnoff C, Caron K, Wang L, Melstrom P, Trivers K. Exposure to secondhand smoke among nonsmokers-United States, 1988-2014. MMWR Morb Mortal Wkly Rep 2018 67(48):1342-1346

22 Wolfson M, McCoy T, Sutfin E. College students' exposure to secondhand smoke. Nicotine Tob Res 2009 11(08):977-984 